\title{
Interplay Between Electronic States and Structural Stability in Cation-Deficient VCoSb, NbCoSb, and TaCoSb Half-Heuslers
}

\author{
Joaquin Miranda ${ }^{1}$ (D) $\cdot$ Thomas Gruhn $^{1}$
}

Received: 8 October 2021 / Accepted: 8 February 2022 / Published online: 3 March 2022

(c) The Author(s) 2022

\begin{abstract}
The effects of the vacancy concentration at the cation site of three half-Heuslers, VCoSb, NbCoSb, and TaCoSb, were studied with a combination of two computational methods: density functional theory and Monte Carlo simulations, both linked by a cluster expansion method. Our density functional method allows us to follow a gap opening in the electronic density of states in $\mathrm{NbCoSb}$ and $\mathrm{TaCoSb}$ as a function of vacancy concentration, starting from a metallic state with the Fermi-level crossing the valence states in the pristine crystal, passing throughout a $p$-type doped behavior, down to a semiconducting state at $20 \%$ of vacancies. In the case of $\mathrm{VCoSb}$, the transition starts from the half-metallic ferromagnetic state, where $\mathrm{VCoSb}$ remains half-metallic until it achieves a semiconductor state at $\mathrm{V}_{0.8} \mathrm{CoSb}$ composition, the transition leading to a magnetic-nonmagnetic crossover. Further increase of vacancies leads to non-polarized in-gap states in $\mathrm{V}_{0.75} \mathrm{CoSb}$, and polarized in-gaps in $\mathrm{Nb}_{0.77} \mathrm{CoSb}$, while $\mathrm{Ta}_{0.75} \mathrm{CoSb}$ recovers a metallic behavior but with an $n$-character. Based on our cluster expansion, we can assert that $\mathrm{Ta}_{0.8} \mathrm{CoSb}$ is slightly more stable than $\mathrm{Nb}_{0.8} \mathrm{CoSb}$, while both are much more stable than $\mathrm{V}_{0.8} \mathrm{CoSb}$. Temperature effects were studied through Monte Carlo simulations. The simulations show that, upon cooling, the ground states are hard to recover, and instead metastable states are formed. The vacancy arrangements were scrutinized with the help of suitable order parameters for the lattice vacancy occupation.
\end{abstract}

Keywords Defective half-Heuslers · vacancies · thermoelectrics $\cdot$ cluster expansion (CE) $\cdot$ Monte Carlo $\cdot$ DFT $\cdot$ correlated disorder

\section{Introduction}

Point defects may appear spontaneously during the manufacturing of a material, ${ }^{1}$ and, despite its misleading name, they are sometimes quite desirable in many branches of material science. When targeting specific physical properties, controlled and selectively induced defects can be used for introducing or tuning desired physical properties. ${ }^{2}$ For instance, point defects play a key role in the field of transparent semiconductors and, ${ }^{3-5}$ recently, it was proposed that vacancies could provide an alternative to obtain the long sought-after $p$-type transparent semiconductor. ${ }^{6}$ In half-Heusler $(\mathrm{HH})$ thermoelectrics, based on semiconductor materials, the understanding and judicious selection of defects has also been beneficial to the performance of materials. ${ }^{7-10}$

Joaquin Miranda

miranda.joaquin@gmail.com

1 Biomaterials, Universität Bayreuth,

Prof.-Rüdiger-Bormann-Str. 1, 95447 Bayreuth, Germany
In thermoelectric materials, the presence of vacancy defects can help to tune the power factor or/and the lattice thermal conductivity to deliver a higher figure-of-merit (ZT). Thus, one finds reports of vacancies assisting the desired reduction of the lattice thermal conductivity in $\mathrm{CuGaTe}_{2},{ }^{11}$ while in $\mathrm{SnTe}-\mathrm{In}_{2} \mathrm{Te}_{3},{ }^{12} \mathrm{Ge}_{9} \mathrm{Sb}_{2} \mathrm{Te}_{12},{ }^{13}$ and $\beta-\mathrm{Zn}_{4} \mathrm{Sb}_{3},{ }^{10}$ the presence of vacancies simultaneously improves the electrical properties and reduces the lattice thermal conductivity. In these improved thermoelectric examples, as in most cases in the field of thermoelectricity, the target was materials with good semiconducting behavior. What we will discuss in this paper is a different strategy in the use of vacancies to control electronic properties in materials, particularly within the family of HH. We present a way to turn a metal (semi-metal) into a semiconductor as well as the possibility of inducing intrinsic $n$ - or $p$-doping via vacancies.

The idea has prevailed that one should first select those HHs which meet 18-valence electron count (VEC) criteria, ${ }^{14-22}$ and then improve their electronic and phonon characteristics. This approach has shown to be useful in $\mathrm{HH}$ 
intermetallic compounds, which are among the thermoelectric material classes that have steadily improved their $Z T$, already achieving values of $1.5 .{ }^{23}$ However, this view was challenged following $Z T$ measurements in $19 \mathrm{VEC} \mathrm{HH}$ at $973 \mathrm{~K}$ with $Z T$ $=0.4$ for $\mathrm{NbCoSb}^{24}$ and $Z T=0.5$ for the $\mathrm{Nb}_{0.44} \mathrm{~V}_{0.44} \mathrm{Ta}_{0.12}$ CoSb alloy. ${ }^{25} \mathrm{~A}$ remarkable discovery ${ }^{26}$ in cation-defective $\mathrm{HH}$ increased $\mathrm{ZT}$ to 0.9 at $1123 \mathrm{~K}$ in $\mathrm{Nb}_{0.83} \mathrm{CoSb}$. In these new materials, their hallmark is the structural stability found at near $20 \%$ of vacancy concentration and optimal ZT slightly above this concentration ${ }^{27,28}$ with the presence of a semiconducting gap. To explain this emergent feature, one should think of $\mathrm{NbCoSb}$ as a heavily doped semiconductor, rather than as a metal. ${ }^{29}$ Based on these observations, a modification of the 18-VEC rule seems more appropriate to predict the formation of stability in a broader range of $\mathrm{HH}$ composition, which includes defects. ${ }^{30}$ The new criterion uses the valence band electronic configuration, which should amount to zero to achieve a semiconductor phase. This novel formulation opens the opportunity to a new route to obtain a semiconductor phase via the manipulation not only of vacancies but also of interstitial and substitutional defects.

On the other hand, cluster expansion (CE) methods have played a very decisive role in computationally corroborating the structural stability at near $20 \%$ of vacancy concentration. $^{26,27,31}$ The aforementioned work showed that CE can handle very well a considerable amount of defects in $\mathrm{HH}$. More recently, ${ }^{32}$ and quite remarkably, Monte Carlo methods based on very simplified models of interaction have been able to reproduce some of the distinctive features seen in electron diffraction patterns of defective $\mathrm{HH}$ systems.

In the light of these discoveries, we decided to scrutinize three 19-VEC HH within a range of vacancy concentrations $(x)$ which simultaneously stabilize the materials and tune their electronic transport properties. We investigate three isoelectronic $\mathrm{HH}$ with formula $M_{1-\mathrm{x}} \mathrm{CoSb}(M=\mathrm{V}, \mathrm{Co}, \mathrm{Sb})$. For that, we employ a $\mathrm{CE}$ which has previously been successfully applied to HHs. ${ }^{7,33,34}$ First, we will detail the transition from a metallic state to semiconductor states triggered by the introduction of vacancies at the $M$ site. We show that the vacancy concentration regulates the transition and makes the materials behave effectively as a doped semiconductor, with the possible ability to set an $n$ or $p$ character for concentrations slightly below or above $x=0.2$, respectively. In the second part, we show how the vacancies self-organize in a particular fashion to minimize the formation energy of the structures.

\section{Cluster Expansion and DFT Analysis}

\section{Methodology}

A Heusler material with formula $X Y_{2} Z$ consists of four interpenetrating fcc sub-lattices, $A, B, C$, and $D$ (see Fig. 1).
The sub-lattices include the points $(0,0,0),(0.25,0.25,0.25)$, $(0.5,0.5,0.5)$, and $(0.75,0.75,0.75)$, which are occupied by $Y, X, Y^{\prime}$ and $Z$ atoms, respectively. In the $X Y Z \mathrm{HH}$, or so-called sub-Heuslers, only three of the four sub-lattices are occupied, $A, B$, and $D$-see the cubic unit cell shown in Fig. 1-while the $C$ sub-lattice is left empty. The HH structure has a $F \overline{4} 3 m$ symmetry. We model three HHs, namely $\mathrm{VCoSb}, \mathrm{NbCoSb}$, and $\mathrm{TaCoSb}$, and study the introduction of vacancies (Vac) at the $B$ sub-lattice occupied by atoms ( $M$ $=\mathrm{V}, \mathrm{Nb}, \mathrm{Ta})$. The sub-lattice $B$ is filled with a fraction $1-x$ of $M$ atoms, and the other two sub-lattices, $A$ and $D$, remain filled with atoms $\mathrm{Co}$ and $\mathrm{Sb}$, respectively.

We use the $\mathrm{CE}$ technique in which the formation energies are calculated with density functional theory (DFT) for a set of $M_{1-\mathrm{x}} \mathrm{Vac}_{\mathrm{x}} \mathrm{CoSb}$ structures with a different number of vacancies and atomic arrangements. Here we use the notation $M_{1-\mathrm{x}} \operatorname{Vac}_{\mathrm{x}} \mathrm{CoSb}$ to explicitly indicate that a vacancy of a $M$ type atom is treated as a pseudo-atom. These formation energies of the structure are then approximated by a function that is expanded in small clusters within active sub-lattices. It means that besides the on-site energy of a single atom occupying a sub-lattice, the subsequent orders expanding the function include clusters of an increasing number of sub-lattice sites: tuples of two sites, sets of three sites, and so on. The largest distance between two sub-lattice points defines the size of the cluster. The treatment of vacancies as pseudo atoms allows us to compute effective interactions among atoms and vacancies. The active sites are the positions defined by the equivalent points of symmetry for the $B$-sub-lattice where $M$ atoms or vacancies are hosted. ${ }^{35}$ By calculating DFT energies for new configurations and adapting the $\mathrm{CE}$, the process is iterated and the formula for the formation energy is improved. ${ }^{36}$ The $\mathrm{CE}$ is considered satisfactory when the tested configurations provide a suitably low value for the so-called cross-validation (CV) score (Table I).

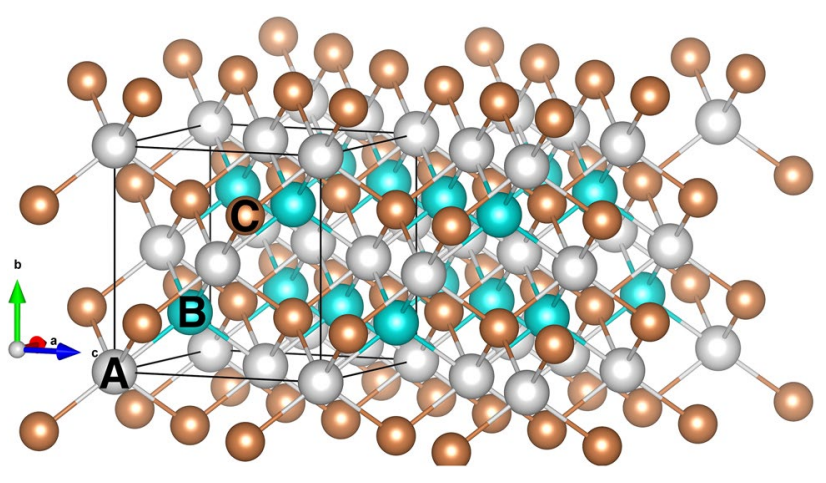

Fig. 1 (Color online) (a) $2 \times 2 \times 1 \mathrm{HH}$ cubic cell with formula $M \operatorname{CoSb}(M=\mathrm{V}, \mathrm{Nb}, \mathrm{Ta}), M$ atoms are colored in cyen, $\mathrm{Co}$ and $\mathrm{Sb}$ atoms are in gray and brown, respectively. The black frame highlights one single cubic cell. The sub-lattices $A, B$ and $D$, together with the empty $C$ sub-lattice (not shown), define the primitive unit cell. 
For the DFT calculations, we used the projector augmented wave method ${ }^{37}$ as implemented in the Vienna ab initio simulations package. ${ }^{38,39}$ The energy cut-off for the expansion of the electronic wave function in plane waves was set to $390 \mathrm{eV}$, while the type of exchange-correlation functional chosen was the Perdew-Burke-Ernzerhof generalized gradient approximation; an $8 \times 8 \times 8$ Monkhorst pack $k$-mesh for the Brillouin integration was selected. Magnetic effects were considered throughout the spin-polarized electronic DOS within the local spin density approximation. Structural optimization was taken into account by allowing the relaxation of the unit cell, ion positions, and lattice parameters. Any force larger than $6 \mathrm{meV} / \AA$ was relaxed with a maximum of 12 ionic steps. Energies below $0.1 \mathrm{meV}$ were used as a self-consistency criterion to stop the calculations. In this work, we obtained a score of CV $=0.061,0.047$, and
0.060 , using 103, 216, and 164 structures for $\mathrm{V}-, \mathrm{Nb}-$, and Ta-based systems, respectively.

\section{Results}

Figure 2a-c shows the DFT energies calculated for the three $\mathrm{HH}$ systems, all of them presenting a minimum energy at $20 \%$ of vacancy concentration $(x=0.2)$. The structure with the lowest energy corresponds to a unit-cell with 19 atoms (5 Co atoms, $5 \mathrm{Sb}$ atoms, and $4 M$ atoms, see the Supplemental Material for a cif file). Henceforth, we use the notation $M_{0.86}$ and $M_{0.83}$, for systems with vacancy concentrations of $1 / 7$ and $1 / 6$, respectively. In all three $M C$ OSb systems, the lowest energy atomic configuration also corresponds to the same unit cell; however, among them, the $\mathrm{V}_{0.8} \mathrm{CoSb}$ has the highest energy of formation, $-0.48 \mathrm{eV}$, while $\mathrm{Nb}_{0.8} \mathrm{CoSb}$

Table I Structural parameters, coordination number $\operatorname{Co} M_{\mathrm{n}}$, and structural energies calculated by DFT

\begin{tabular}{|c|c|c|c|c|c|c|c|c|c|c|c|c|}
\hline$M_{1-\mathrm{x}} \operatorname{Vac}_{\mathrm{x}} \mathrm{CoSb}$ & $a(\AA)$ & $b(\AA)$ & $c(\AA)$ & $\alpha\left(^{\circ}\right)$ & $\beta\left(^{\circ}\right)$ & $\gamma\left({ }^{\circ}\right)$ & Vol. $\left(\AA^{3}\right)$ & $\mathrm{Co} M_{1}$ & $\mathrm{CoM}$ & $\mathrm{CoM}_{3}$ & $\mathrm{Co} M_{4}$ & Energy $(\mathrm{eV})$ \\
\hline$S 4-\mathrm{Ta}_{0.8} \mathrm{CoSb}$ & 14.95 & 14.95 & 14.95 & 163.75 & 163.75 & 23.06 & 261.90 & 0 & 1 & 2 & 2 & -0.65 \\
\hline$S 2-\mathrm{Ta}_{0.8} \mathrm{CoSb}$ & 7.25 & 7.25 & 10.26 & 118.27 & 118.27 & 33.55 & 259.02 & 0 & 1 & 3 & 1 & -0.73 \\
\hline$G-\mathrm{Ta}_{0.8} \mathrm{CoSb}$ & 7.22 & 7.22 & 7.22 & 99.74 & 99.74 & 131.41 & 257.80 & 0 & 0 & 4 & 1 & -0.82 \\
\hline$S Q S 3-\mathrm{Nb}_{0.8} \mathrm{CoSb}$ & 7.30 & 9.44 & 12.64 & 72.59 & 73.18 & 82.41 & $795.26(265.0)$ & 0 & $2(0.6)$ & $7(2.3)$ & $6(2)$ & -0.49 \\
\hline$S Q S 2-\mathrm{Nb}_{0.8} \mathrm{CoSb}$ & 11.05 & 11.05 & 7.25 & 96.34 & 96.34 & 141.71 & $516.90(258.4)$ & 0 & $2(1.0)$ & $4(2.0)$ & $4(2)$ & -0.62 \\
\hline$S Q S 1-\mathrm{Nb}_{0.8} \mathrm{CoSb}$ & 7.23 & 7.23 & 10.21 & 118.10 & 118.10 & 33.57 & 257.36 & 0 & 1 & 3 & 1 & -0.64 \\
\hline $\mathrm{Nb}_{0.8} \mathrm{CoSb}$ & 7.25 & 7.25 & 7.25 & 99.74 & 99.74 & 131.43 & 261.03 & 0 & 0 & 4 & 1 & -0.72 \\
\hline$S 1-\mathrm{V}_{0.2} \mathrm{CoSb}$ & 7.08 & 7.08 & 7.08 & 13.66 & 13.66 & 13.66 & 242.02 & 1 & 0 & 1 & 3 & -0.15 \\
\hline$G-\mathrm{V}_{0.2} \mathrm{CoSb}$ & 7.11 & 7.10 & 7.08 & 99.77 & 99.77 & 131.58 & 243.46 & 0 & 0 & 4 & 1 & -0.46 \\
\hline
\end{tabular}

Lines two to four are different atomic arrangements at the same composition $\mathrm{Ta}_{0.8} \mathrm{CoSb}$ (the $S$ label correspond to higher energy structures and $G$ to the lowest energy; their density of states (DOS) are shown in Fig. 4).

Lines five to eight are for three SQS structures at the same composition $\mathrm{Nb}_{0.8} \mathrm{CoSb}$ and the lowest energy state (see discussion in "Results" section).

The last two lines are for the only two structures created by the $\mathrm{CE}$ for $\mathrm{V}_{0.8} \mathrm{CoSb}$.

All cells are composed by 14 atoms ( $4 M, 5 \mathrm{Co}$ and $5 \mathrm{Sb}$ ).

Only $S Q S 3-\mathrm{Nb}_{0.8} \mathrm{CoSb}$ and $\mathrm{ISQS} 2-\mathrm{Nb}_{0.8} \mathrm{CoSb}$ are composed by 29 (9 M, $10 \mathrm{Co}$ and $\left.10 \mathrm{Sb}\right)$ and 44 atoms (14 M, $15 \mathrm{Co}$ and $\left.15 \mathrm{Sb}\right)$, respectively. For a better comparison, in the parentheses are values normalized with respect a cell of 14 atoms.
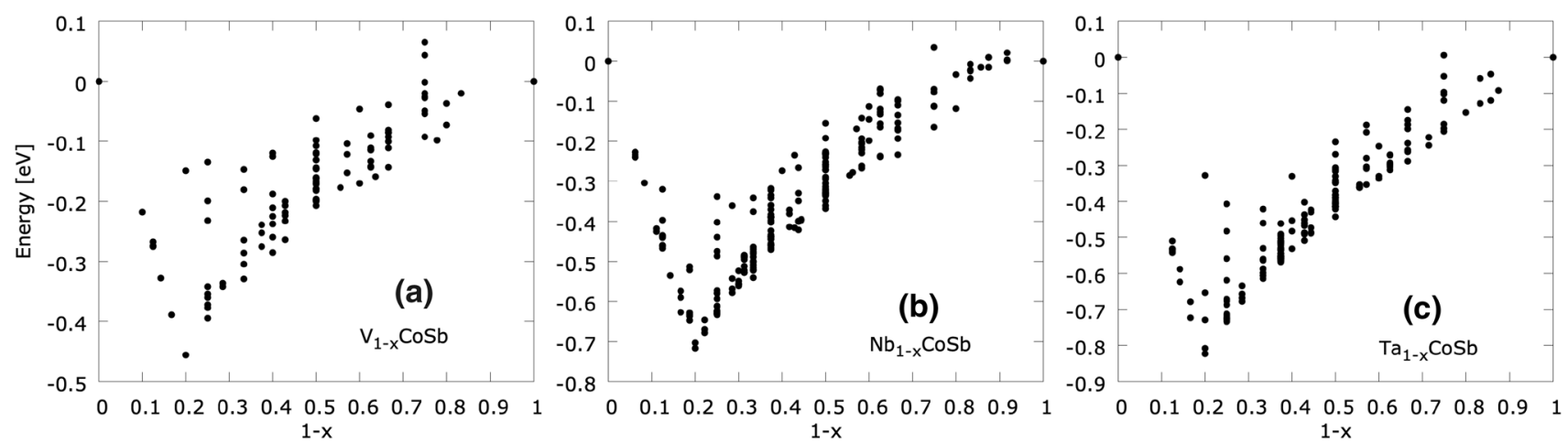

Fig. 2 DFT energies calculated for the structures in the HH systems: (a) $\mathrm{V}_{1-\mathrm{x}} \mathrm{CoSb}$, (b) $\mathrm{Nb}_{1-\mathrm{x}} \mathrm{CoSb}$, and $\odot$ ) $\mathrm{Ta}_{1-\mathrm{x}} \mathrm{CoSb}$. 
and $\mathrm{Ta}_{0.8} \mathrm{CoSb}$ are $-0.73 \mathrm{eV}$ and $-0.82 \mathrm{eV}$, respectively, indicating that the latter two are more stable. Notice that other unit cells with $20 \%$ of vacancies were also created during the $\mathrm{CE}$ procedure, but they have higher energies (see Discussion below).

From Fig. 2, we can also see that $\mathrm{V}_{1-\mathrm{x}} \mathrm{CoSb}$ has a lower affinity to host vacancies, as is deduced from the slope, $\mathrm{d} E /$ $\mathrm{d} x$, formed between the two points $(E=0, x=0)$ and $(E=$ $0.41 \mathrm{eV}, x=0.2)$. It has a less negative slope within the $x$ range $[0,0.2]$ than the slopes formed for $\mathrm{Nb}_{1-\mathrm{x}} \mathrm{CoSb}$ and $\mathrm{Ta}_{1-\mathrm{x}} \mathrm{CoSb}$. The lattice parameter for the most stable structures below $x \leq 0.2$ listed in Table II shows that vacancies reduce the volume of the unit cells monotonously. For a given $x, \mathrm{~V}_{1-\mathrm{x}} \mathrm{CoSb} \mathrm{HHs}$ present smaller lattice parameters than $\mathrm{Nb}_{1-\mathrm{x}} \mathrm{CoSb}$ and $\mathrm{Ta}_{1-\mathrm{x}} \mathrm{CoSb}$.

We should point out that, at a very high concentration of vacancies, our model may not describe well the procedure of the synthetization of the materials, where, for instance, secondary structures like $\mathrm{Nb}_{3} \mathrm{Sb}$ are observed. Notice that the complete removal of $M$ atoms, $x=1$ point in Fig. 2, corresponds to the zinc blende structure for the CoSb binary system. Nevertheless, we expect that our model can describe concentrations below the $30 \%$ limit, where the $\mathrm{HH}$ phases strongly dominate the material.

The higher stability of the structures in $M_{1-\mathrm{x}} \mathrm{CoSb}$, as more vacancies are introduced is reflected in the electronic DOS, especially in the gaps seen at negative energies for the parental structures, which are shifted towards energies closer to the Fermi level until they form a semiconducting gap at $x=0.2$. We follow this process in Fig. 3. Without vacancies, $\mathrm{NbCoSb}$ and TaCoSb behave like metals, while VCoSb is a semi-metal. At near $17 \%$ of vacancies, the three systems reduce their DOS to around the Fermi level. In this range of concentrations, the DOS structures are similar to those of $n$-doped semiconductors. Note that $\mathrm{V}_{0.83} \mathrm{CoSb}$ is still semimetallic; in addition, its Fermi level is positioned slightly

Table II Lattice parameters in $\AA$ for some selected stable structures for the three $M_{\mathrm{x}} \mathrm{CoSb}(M=\mathrm{V}, \mathrm{Nb}$, Ta) HHs within the $0.0 \leq x \leq 0.2$ range

\begin{tabular}{llll}
\hline$M_{\mathrm{x}} \mathrm{CoSb}$ & \multicolumn{3}{l}{} \\
\cline { 2 - 4 } & $\mathrm{V}$ & $\mathrm{Nb}$ & $\mathrm{Ta}$ \\
\hline$M \mathrm{CoSb}$ & 5.8151 & 5.9650 & 5.9554 \\
$M_{0.890} \mathrm{CoSb}$ & 5.7789 & 5.9352 & - \\
$M_{0.875} \mathrm{CoSb}$ & 5.7753 & 5.9318 & 5.9161 \\
$M_{0.86} \mathrm{CoSb}$ & 5.7672 & 5.9359 & 5.9242 \\
$M_{0.833} \mathrm{CoSb}$ & 5.7450 & 5.9133 & 5.8989 \\
$M_{0.800} \mathrm{CoSb}$ & 5.7361 & 5.8793 & 5.8869 \\
\hline
\end{tabular}

Experimental values reported by Kaczmarska et al..$^{40}$ for two parental structures are: $5.802 \AA$ and $5.904 \AA$ for $\mathrm{NbCoSb}$ and $\mathrm{VCoSb}$, respectively. deeper into the conduction band than the corresponding Fermi levels of $\mathrm{Nb}_{0.83} \mathrm{CoSb}$ and $\mathrm{Ta}_{0.83} \mathrm{CoSb}$. Furthermore, the gap of $\mathrm{V}_{0.83} \mathrm{CoSb}$ is smaller than those of $\mathrm{Nb}_{0.83} \mathrm{CoSb}$ and $\mathrm{Ta}_{0.83} \mathrm{CoSb}$. A semi-conducting gap is fully formed at $20 \%$ of vacancies, with the Fermi level lying exactly at the edge of the valance band for the three systems. The gap widths are $0.72 \mathrm{eV}, 1.0 \mathrm{eV}$, and $1.12 \mathrm{eV}$ for $\mathrm{V}_{0.8} \mathrm{CoSb}, \mathrm{Nb}_{0.8} \mathrm{CoSb}$, and $\mathrm{Ta}_{0.8} \mathrm{CoSb}$, respectively. One can see that $\mathrm{V}_{0.8} \mathrm{CoSb}$ has lost all traces of magnetism, as there is no dis-balance among the spin-up and spin-down electrons, nor in the conduction electrons, nor in the valance bands. The fact that, in the metallic states, electrons from the cations which are located in the conduction band participate in the bonding, but weakly, ${ }^{20}$ via the overlapping with the Co atoms (see red and green lines in the DOS of Fig. 3) facilitates the formation of vacancies when the chemical bonds break.

A further increase of the vacancies shifts the Fermi level into the valance bands for $\mathrm{Ta}_{0.75} \mathrm{CoSb}$, giving it a $p$-semiconducting character. Interestingly, for $\mathrm{V}_{0.75} \mathrm{CoSb}$ and $\mathrm{Nb}_{0.77}$ $\mathrm{CoSb}$ (see Supplementary Material), we observe some electronic states forming in-gap states, separated by $0.12 \mathrm{eV}$ and $0.23 \mathrm{eV}$, respectively, from the valence states. These in-gap states present different spin-polarizations: while the one in $\mathrm{V}_{0.75} \mathrm{CoSb}$ is not polarized, the in-gap states in $\mathrm{Nb}_{0.77} \mathrm{CoSb}$ are fully polarized. In the former case, the net magnetization is 0 , while in the latter, it is $0.12 \mu_{\mathrm{B}}$. Indeed, at $25 \%$ of vacancies, the three compounds present different DOS distributions around the Fermi level. At this vacancy concentration, the in-gap states are seen only in $\mathrm{V}_{0.75} \mathrm{CoSb}$. For $\mathrm{Nb}_{0.75} \mathrm{CoSb}$, the magnetization increases to $0.22 \mu_{\mathrm{B}}$, but the full polarized in-gap of $\mathrm{Nb}_{0.77} \mathrm{CoSb}$ has disappeared (see Figs. S2, S3 and Table S1 of the Supplemental Material); instead, spin-up states appear just below the Fermi energy and the spin-down states just above it. For $\mathrm{Ta}_{0.75} \mathrm{CoSb}$, the electronic states of both spin channels remain practically identical, with a small dis-balance between both types of spins at around $0.4 \mathrm{eV}$ above the Fermi energy.

To understand the differences between the three $M_{0.75}$ $\mathrm{CoSb} \mathrm{HH}$, it is crucial to first observe the peaks of DOS below the Fermi-energy (marked as $P$ and $P^{\prime}$ in Fig. 3 ) at the $M_{0.8} \mathrm{CoSb}$ compositions. Our interpretation is that the extra $5 \%$ of vacancies deplete the states previously lying in $\mathrm{V}_{0.8}$ $\mathrm{CoSb}$ below the Fermi level and close to the $P$ peaks. The new position of the Fermi level for $\mathrm{V}_{0.75} \mathrm{CoSb}$ falls below the peak $P$; importantly, the remaining amount of electron states allows the keeping of an equal population of up- and down-spins which lead to the unpolarized in-gap. In $\mathrm{Nb}_{0.75}$ $\mathrm{CoSb}$, the depletion redistributes the DOS, creating the condition to meet the Stoner criteria at the level of the $P$ energy. An exchange interaction splits the energy of states with different spins, with the states near the Fermi energy being spin-polarized, leading to the ferromagnetic state. In $\mathrm{Ta}_{0.75} \mathrm{CoSb}$, the DOS around the Fermi energy becomes very 

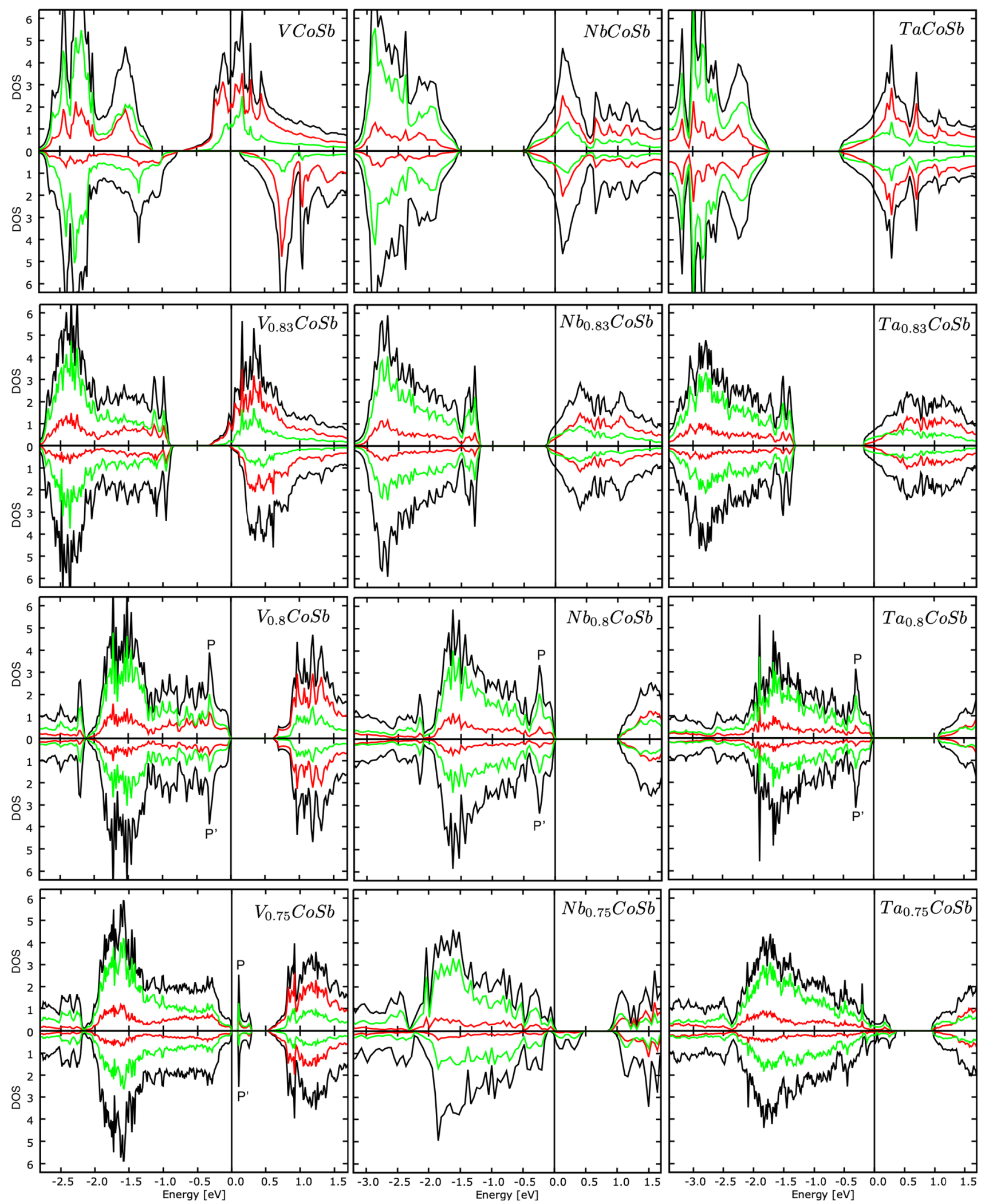

Fig. 3 (Color online) Spin-resolved density of states, plotted in black, for $\mathrm{V}_{1-\mathrm{x}} \mathrm{CoSb}$ (left panels), $\mathrm{Nb}_{1-\mathrm{x}} \mathrm{CoSb}$ (center panels), and $\mathrm{Ta}_{1-\mathrm{x}}$ $\mathrm{CoSb}$ (right panels). The first, second, third and fourth rows corre- spond to $x=0, x \approx 0.17, x=0.20$ and $x=0.25$, respectively. The red lines are the contributions from the cation atoms, and the green lines from the Co atoms. 
flat and, therefore, the Stoner criteria is not fulfilled. We think that the extension of the cation's d-wave function is a decisive factor in producing such a diverse electronic character. The more localized 3d-wave function of $\mathrm{V}$ keeps the DOS narrow but at high enough energies with respect to the Fermi energy to avoid the Stoner condition. Conversely, the broader 5d-wave function of Ta induces a widespread DOS, which also prevents meeting the condition. It is only the right extension provided by the $4 \mathrm{~d}$-wave of $\mathrm{Nb}$ which allows the proper DOS distribution to meet the Stoner criteria.

In addition to the ground states we have discussed above, the CE created other structures with higher energies. These structures can provide information about the expected crystallographic and physical quality of materials obtained by a manufacturing process that does not match the required condition to grow a crystal with the symmetry of the lowest energy structures. Therefore, it is important to address how much the electronic properties of higher energy states can deviate from those seen in the lowest energy state. As an example, we take a look at $\mathrm{Ta}_{0.8} \mathrm{CoSb}$. We plot in Fig. 4 the total DOS of three structures, which are $0.045 \mathrm{eV}, 0.263$ $\mathrm{eV}$, and $0.483 \mathrm{eV}$ higher in energy (energy per $M_{0.8} \mathrm{CoSb}$ formula) than the ground states. We label these three states $S 1, S 2$, and $S 3$, respectively. The two states closer in energy to the ground states still present a semiconducting gap, but with reduced width; thus, for $S 1$ the width is $0.89 \mathrm{eV}$, and for $S 2$ is $0.37 \mathrm{eV}$. In contrast, for $S 3$, the semiconducting gap has closed. Therefore, there is a fair possibility to observe semiconducting gaps smaller than $1.1 \mathrm{eV}$ in $\mathrm{Ta}_{0.8}$ $\mathrm{CoSb}$ grown under techniques that do not allow the highest control to obtain materials with the ground state symmetries. Similarly, for $\mathrm{V}_{0.8} \mathrm{CoSb}$ and $\mathrm{Nb}_{0.8} \mathrm{CoSb}$, a gap remains for structures with energies close to the ground states.

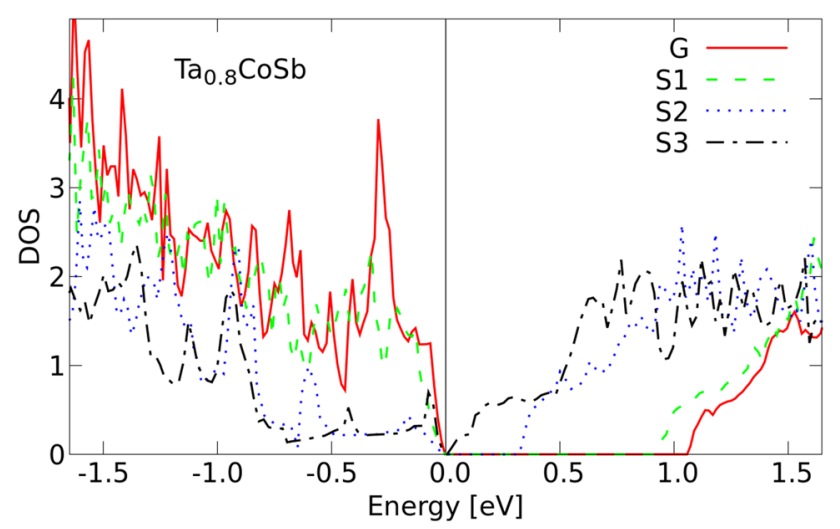

Fig. 4 (Color online) Total DOS for four structures with the same $\mathrm{Ta}_{0.8} \mathrm{CoSb}$ composition but different vacancy distributions. The DOS for the ground state is labeled $G$, the three other structures with higher energy are labeled $S 1, S 2$, and $S 3$. The structures have the following order of formation energy: $E_{\mathrm{GS}}<E_{\mathrm{S} 1}<E_{\mathrm{S} 2}<E_{\mathrm{S} 3}$.
The coordination number formed by the Co and $M(M$ $=\mathrm{V}, \mathrm{Nb}$ ) in the compound can help to explain the differences between the highest and lowest energy structures at a given $M_{0.8} \mathrm{CoSb}$ composition. In principle, the coordination number can take values from zero to four. Henceforth, we denote the number of $M$ atoms bounded to a centered Co as $\mathrm{CoM}_{\mathrm{n}}(n=1,2,3,4)$, where, for instance, $\mathrm{CoM}_{3}$ means the coordination is reduced to three, due to the absence of one $M$ atom. We will use $\mathrm{Co} M_{\mathrm{n}}$ as indicators to compare structural stability. First of all, we notice that the DFT calculations for the same compositions predict higher energies of formation for cells with larger volumes than for cells with smaller volumes (see Table I). Moreover, the high energy cells present a clear tendency to have more $\mathrm{Co} M_{2}$ and $\mathrm{Co} M_{4}$ amounts than the low energy cells. We use these observations to prove that vacancy clustering is energetically not helpful for the stability of cation-deficient HHs. To illustrate our argument, we focus on the lowest and the highest energies of $\mathrm{Ta}_{0.8} \mathrm{CoSb}$ cells discussed in Fig. 4. Thus, for $S 4-\mathrm{Ta}_{0.8} \mathrm{CoSb}$ (energy formation $-0.65 \mathrm{eV}$ ), $\mathrm{Co} M_{2}=1$ and $\mathrm{Co} M_{3}=2, M_{4}=2$, while for $S 2-\mathrm{Ta}_{0.8} \mathrm{CoSb}$ (energy formation $-0.73 \mathrm{eV}$ ), $\mathrm{CoM}_{2}=1$, $\mathrm{Co}_{3}=3$ and $\mathrm{Co} M_{4}=1$. In the lowest energy structure, $G$ $\mathrm{Ta}_{0.8} \mathrm{CoSb}$ (energy formation $-0.88 \mathrm{eV}$ ), $\mathrm{Co} M_{2}=0, \mathrm{Co} M_{3}$ $=4$ and $\mathrm{CoM}_{4}=1$ (the $S 2-\mathrm{Ta}_{0.8} \mathrm{CoSb}$ and $G-\mathrm{Ta}_{0.8} \mathrm{CoSb}$ unit cells and the different $\mathrm{Co} M_{\mathrm{n}}$ coordinations are shown in Fig. $\mathrm{S} 1$ of the Supplementary Material). One infers from this that the simultaneous appearance of two and four coordinations in the higher energy structures is because there are regions that contain close voids formed by the vacancies, while, in the lowest energy cells, the absence of two coordinations but the increased presence of three coordinations is due to the more homogeneous distributions of the void spaces. Hence, this indicates that the proximity of the vacancies forms large volumes, which in turn creates larger instability. Similar tendencies are seen for structures with the $\mathrm{V}_{0.8} \mathrm{CoSb}$ and $\mathrm{Nb}_{0.8}$ $\mathrm{CoSb}$ compositions.

In some high-energy cells, the vacancies arrangements form such disconnected regions that they look structurally very unlikely to occur in practice. That is, for instance, the case of the only high-energy point at the $\mathrm{V}_{0.8} \mathrm{CoSb}$ composition seen in Fig. $2 \mathrm{a}$, and denoted as $S 1-\mathrm{Ta}_{0.8} \mathrm{CoSb}$ in Table $\mathrm{I}$. The vacancies have created single $\mathrm{Co}-\mathrm{V}$ pairs $\left(\mathrm{Co} M_{1}=1\right)$ and a higher amount of $\mathrm{Co}-\mathrm{V}$ coordinations $\left(\mathrm{Co} M_{4}=3\right)$, and these result in the disconnected slaps seen in the extended $S 1-\mathrm{Ta}_{0.8} \mathrm{CoSb}$ presented in Fig. S1d of the Supplementary Material. In contrast, the most stable $M_{0.8} \mathrm{CoSb}$ structures look denser, as observed in the extended versions of the $G$ $\mathrm{Ta}_{0.8} \mathrm{CoSb}$ cell presented in Fig. S1c of the Supplementary Material.

The vacancy arrangement is analyzed in Table III by investigating the superstructures created by the vacancies. At the same time, the data provide information about the self-organization of the vacancies as their concentration 
increases. We use the $I$-shell concept of vacancies separated by an fcc lattice vector, $\mathbf{R}_{\mathrm{i}}$, to count the site occupation, defined as $n_{\mathrm{i}}$ (In Table III, the first three columns give pertinent information for the first nine shells). Due to the fewer number of vacancies, it seems that it is energetically possible at $x=1 / 7$ to accommodate six vacancies in the third shell (two less than for $x=1 / 6$ and $1 / 5$ concentrations), while keeping without vacancies the nearest neighbors (NN), next nearest neighbors (NNN), and the fourth shell. For $x$ $=1 / 6$ and $x=1 / 5$, both concentrations can avoid the highenergy cost of having NN vacancies. At $x=1 / 5$, we see the first composition, $M_{0.8} \mathrm{CoSb}$, presenting NNN vacancies as a result of the large number of vacancies introduced. In the next section, we will define an order parameter that characterizes these superstructures. Moreover, we will test the

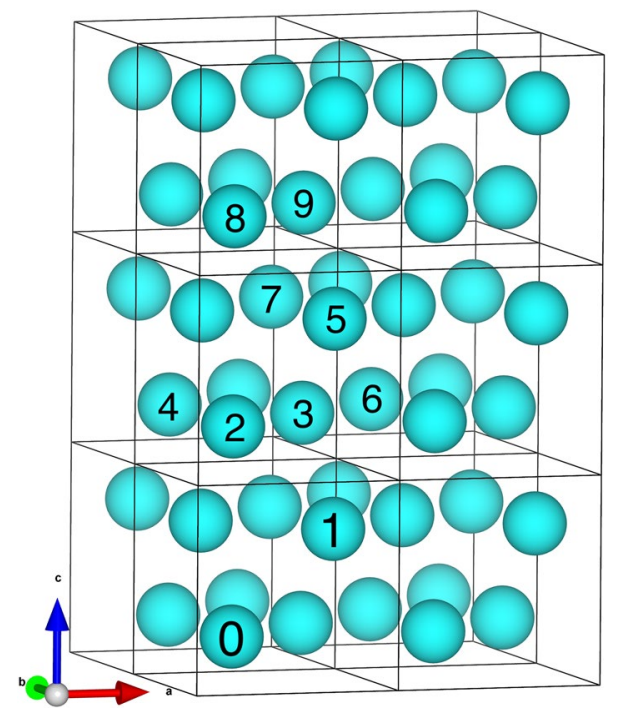

Fig. 5 (Color online) Auxiliary structure displaying only the fcc sublattice $B$ of a $2 \times 2 \times 3 \mathrm{HH}$ cell. The enumerated sites are located in the $I$-shell around the $B$-sub-lattice, which is labeled 0 . stability of the ground states against thermal fluctuations and follow their order-disorder transition. In Fig. 5, we provide an auxiliary cell to enumerate the atoms which are located in the $I$-shell.

\section{Monte Carlo (MC) Simulations}

\section{Methodology}

MC simulations using the interaction coefficients (ICs) of the $\mathrm{CE}$ can access the configurational energy of larger atomic configurations, which in turn allows us to analyze collective behaviors as well as to study temperature effects. This is achieved by utilizing the Metropolis algorithm, ${ }^{41}$ which exchanges the positions of atoms and vacancies at a given temperature. To this end, we perform MC simulations in the canonical ensemble to study order-disorder transitions at finite temperatures. At each temperature, we let the code run for a certain $\mathrm{MC}$ time and a certain number of MC steps until the energy has achieved a precision of $0.001 \mathrm{eV}$. We use systems which include active lattice sites, $N_{\text {act }}$, originated from the $B$ sub-lattice. Simulations with a fraction $x$ of vacancies are performed with a fixed number $N_{\text {Vac }} \simeq x N_{\text {act }}$ of vacancies and number $N_{\mathrm{M}}=N_{\mathrm{act}}-N_{\mathrm{Vac}}$ of $M$ atoms. In the simulations, one MC cycle consists of $N_{\text {act }}$ single steps, in which one active site for an $M$ atom and one for a vacancy are randomly chosen. The old configuration is changed into a trial configuration by swapping the atom and vacancy of the two sites. Following the Metropolis algorithm, ${ }^{41}$ the trial configuration is accepted or rejected. The number of active lattice sites used for the simulations was between 171,500 and 274,625 .

We should confirm that, in our canonical procedure, once the numbers of $\mathrm{Nb}$ atoms and vacancies are fixed, their energy contribution is simply subtracted. With the semigrand canonical ensemble, which is can be employed in the
Table III Occupation of the $I$-shell at $200 \mathrm{~K}$ for the $M_{0.86}$ $\mathrm{CoSb}, M_{0.83} \mathrm{CoSb}$ and $M_{0.80}$ $\mathrm{CoSb}$ ground states; denoted, respectively, as $n_{\mathrm{i}}(1 / 7), n_{\mathrm{i}}(1 / 6)$ and $n_{\mathrm{i}}(1 / 7)$

\begin{tabular}{rrllllll}
\hline$I$ & $Z_{\mathrm{i}}$ & $\left|\mathbf{R}_{\mathrm{i}}\right| \mathrm{L}$ & $n_{\mathrm{i}}(1 / 7)$ & $n_{\mathrm{i}}(1 / 6)$ & $n_{\mathrm{i}}(1 / 5)$ & $O_{\mathrm{i}}\left(\mathbf{R}_{\mathrm{i}}, 1 / 6\right)$ & $O_{\mathrm{i}}\left(\mathbf{R}_{\mathrm{i}}, 1 / 5\right)$ \\
\hline 1 & 12 & 0.707 & 0 & 0 & 0 & -0.200 & -0.250 \\
2 & 6 & 1.000 & 0 & 0 & 2 & -0.200 & 0.167 \\
3 & 24 & 1.224 & 6 & 8 & 8 & 0.200 & 0.167 \\
4 & 12 & 1.414 & 0 & 2 & 0 & 0 & -0.250 \\
5 & 24 & 1.581 & 6 & 4 & 4 & 0 & -0.042 \\
6 & 8 & 1.732 & 2 & 0 & 0 & -0.200 & -0.250 \\
7 & 48 & 1.870 & 6 & 4 & 16 & -0.100 & 0.167 \\
8 & 6 & 2.000 & 0 & 2 & 2 & 0.200 & 0.167 \\
9 & 36 & 2.121 & 0 & 10 & 0 & 0.130 & -0.250 \\
\hline
\end{tabular}

The second column lists the number of sites in a given shell. The third column lists the relative distance between the shell and the centered vacancy, the $L$ units correspond to the lengths of the $M_{1-\mathrm{x}} \mathrm{CoSb}$ unit cells $(x=1 / 7,1 / 6,1 / 5)$, and the last two columns are order parameters (Eq. 1) for $M_{0.83} \mathrm{CoSb}$ and $M_{0.80}$ $\mathrm{CoSb}$ at $200 \mathrm{~K}$ 
ATAT software package, it would be possible to find the ratio of equilibrium between $\mathrm{Nb}$ atoms and their vacancies at a given temperature. This is because, during the implementation of CE, the chemical potentials are also calculated.

To quantify the degree of ordering at a given vacancy concentration and temperature $(T)$, we define an order parameter (OP) ${ }^{42-44}$ for two vacancies separated by an fcc lattice vector $\mathbf{R}_{\mathrm{i}}$ as:

$O\left(R_{\mathrm{i}}, x ; T\right)=\frac{P(i, x ; T)-x}{1-x}$,

where $I$ is the coordinate shell of the lattice vector centered around a vacancy. $P(I, x, T)$ is the probability at temperature $T$ of such a shell to be occupied by vacancies, and we calculate it by means of

$P\left(R_{\mathrm{i}}, x ; T\right)=\frac{C_{\mathrm{i}} / Z_{\mathrm{i}}}{x N_{\mathrm{act}}}$.

Here $C_{\mathrm{i}}$ is the number of vacancies counted in the $I$-shell during an $\mathrm{MC}$ step, and $Z_{\mathrm{i}}$ is the coordination number of that shell. The form of Eq. 1 measures the deviation of vacancy occupation with respect to a statistical average distribution: if the OP matches this average, it is zero for an average occupation. If $O\left(R_{\mathrm{i}}, x ; T\right)$ takes negative values, this indicates that vacancies avoid being placed in the $I$-shell relative to a centered vacancy, while if they are positive, there is a tendency for that shell to be filled by vacancies. If $O\left(R_{\mathrm{i}}, x ; T\right)$ is positive, the vacancy concentration in the $I$-shell is larger than average. We can consider the combination of values taken by the $I$-th order parameter of a composition, $O\left(R_{\mathrm{i}}, x ; T\right)$, with $I=1,2, \ldots 9$, as real space fingerprints of the structures we will discuss.

We highlight some relevant features of atomic-vacancy arrangements extracted from the first OPs of the ground states without the effects of the temperature in Table III. For instance, with $17 \%$ of vacancies, one has $O\left(R_{\mathrm{i}}, x ; T\right)=-0.2$, indicating a scarcity of vacancies in the $\mathrm{NN}$ and $\mathrm{NNN}$ shells, while, for $I=4,5$, values close to 0 indicate average vacancy concentration. Here, it is worth mentioning that these two last vacancy occupations coincide with a random homogenous distribution, although they are intrinsically ordered. Increasing the amount of vacancies to $20 \%$ leads to an OP for $\mathrm{NN}$ without vacancies to a value of -0.25 .

\section{Results}

We performed MC simulations using the ICs obtained by the fitting of $\mathrm{CE}$ to the number of structures created in the "Cluster Expansion and DFT Analysis" section. For the $\mathrm{MC}$ simulations, we restricted ourselves to $\mathrm{Nb}_{\mathrm{x}} \mathrm{CoSb}$. The other two materials behave very similarly. We followed the change in energy as we decreased the temperature in an initial structure formed by the random distribution of vacancies. The initial structure was created by applying the MC simulations at $4000 \mathrm{~K}$, an artificial state, just chosen for numerical reasons in order to achieve a completely disordered initial state. We investigated systems for $x=0.8$ and close by concentrations. It turned out that, while the CE obtained from the standard ATAT process is well suited to study ground states, it does not perform well at higher temperatures. In fact, the internal energy increased at a distinctly too high energy, indicating that higher energy states are not satisfactorily represented. The reason is that the ATAT procedure had found only a very few higher energy states for $x=0.8$. Therefore, we refined the description of the $\mathrm{Nb}_{0.8}$ CoSb composition by instructing the CE to consider highenergy disorder structures throughout the inclusion of special quasi-random structures (SQS). We create 14 supercells with the help of the SQS algorithm (see Zunger et al. $\mathrm{in}^{45}$ ), and subsequently, we applied the CE to the 230 extended sets of $\mathrm{Nb}_{\mathrm{x}} \mathrm{CoSb}$ structures, which gave $\mathrm{CV}=0.043$ during the fitting procedure.

Once we have obtained the ICs for each set, we can analyze the energy dependence with the temperature (the results are displayed in Fig. 6a). We notice an important modification to the curve without SQS structures (labeled as No-SQS in Fig. 6a): The new curves present lower phase transition temperatures than the corresponding energy curves for $\mathrm{MC}$ with no SQS supercells. We should mention that, in previous $\mathrm{CE}^{26}$ trying to describe the same system, $-0.605 \mathrm{eV}$ of energy was reported in the low-temperature regime, which is higher than the energy we found, indicating that our MC simulation has been able to find a lower energy arrangement of vacancies than the states in Ref. 26.

We looked into the order parameters and MC snapshots to collect information about the different effects of temperature on the atomic ordering. The temperature changes of OPs for the MC simulation (Fig. 6b) follow a similar trend, as its corresponding energy curve shows in Fig. 6a; importantly, the regions where they change their slope are within the range where the energies show the transition temperatures. The OPs bring help to reveal information about the preferred arrangement of the vacancies. The -0.2 value for $\mathrm{NN}$ vacancies at low temperature indicates a strong reduction in the likelihood of finding vacancies at these lattice positions. The two next reduced likelihoods correspond to the fourth and sixth shells. On the other hand, the OP shows that vacancies tend to be located at the third shell, while some traces of medium-ranged order are developed in the eighth shell.

The snapshots complement the information reported by the OPs. The ordering created in $\mathrm{Nb}_{0.8} \mathrm{CoSb}$ is shown in the snapshot of Fig. 7. A close look at it reveals three main characteristics: (1) the system is not homogeneous, rather it presents domains with well-defined patterns; some domains being separated by defective or weakly ordered states; (2) 


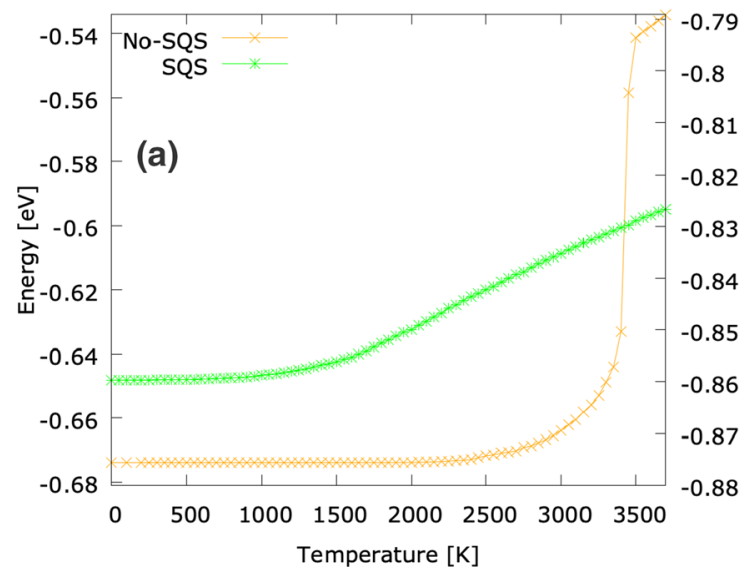

Fig. 6 (Color online) (a) Temperature energy dependence in $\mathrm{Nb}_{0.8}$ $\mathrm{CoSb}$ when no SQS supercells are included in CE (orange curve, right axis) and when the SQS supercells are taken into account (green

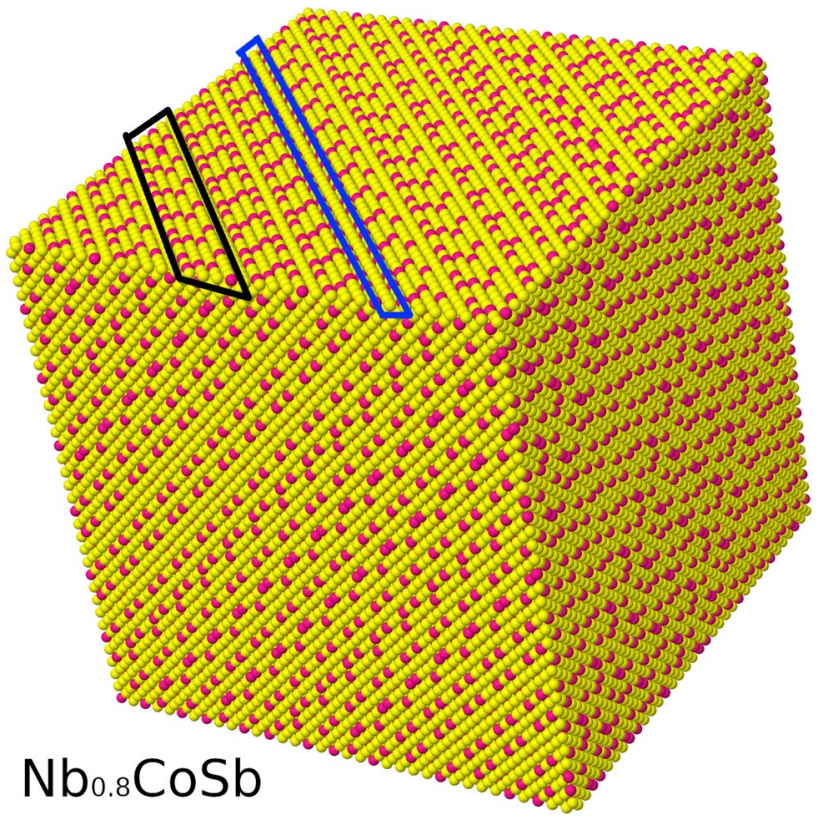

Fig. 7 (Color online) Snapshot showing at $300 \mathrm{~K}$ the atom-vacancy arrangements in $\mathrm{Nb}_{0.8} \mathrm{CoSb}$ obtained by $\mathrm{MC}$ simulations.

the structures differ from those of the ground state; and (3) the vacancies form distinct patterns along the $\{110\}$ direction, or equivalent orientations. The patterns change from line to line in a non-obvious way. In general, one finds either one single vacancy or a pair of $\mathrm{NN}$ vacancies every second or third $\mathrm{Nb}$ atom. The black contour depicts a set of such arrangements in Fig. 7. Note also the lines formed by $\mathrm{Nb}$ atoms which run parallel to the lines of the vacancy patterns (see, for instance, the region marked in blue in Fig. 7). Previous studies applying the CE expansion have also shown that two vacancies prefer to be located in the third shell. ${ }^{31}$

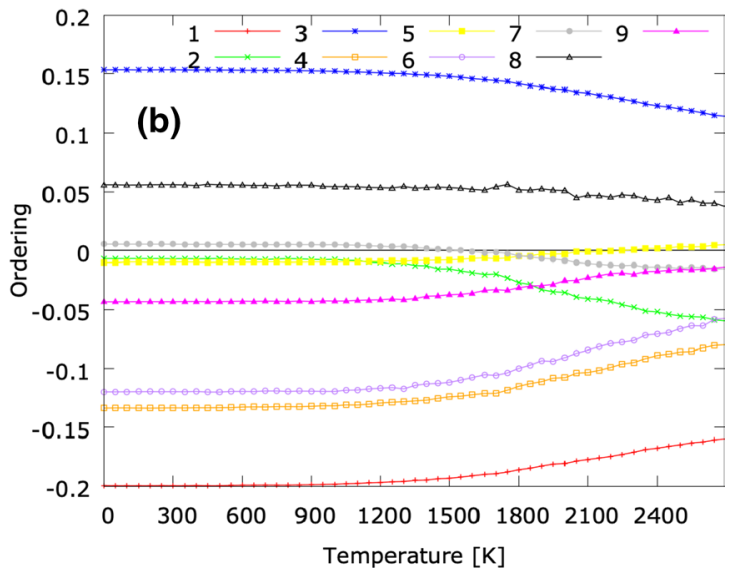

curve, left axis), (b) order parameters in $\mathrm{Nb}_{0.8} \mathrm{CoSb}$ when configurational entropy is considered. Numbers label the corresponding atomic shells.

However, based on the reported snapshots and analysis of the real-space distribution of vacancies, our MC simulations seem to develop higher medium-range ordering. Similarly, with the MC snapshots plotted in Ref. 26, it is difficult to conclude whether or not any type of short- to mediumrange ordering is present. Perhaps these observations can be explained because, in our MC simulations, we used much larger systems. And, instead of truncating or selectively removing terms of the $\mathrm{CE}$, we used the full $\mathrm{CE}$, which may lead to lower energy states corresponding to higher ordering, as we commented at the end of the second paragraph of this section for Fig. 6 a.

\section{Conclusions}

With the help of a cluster expansion method, we studied the structural formation in $\mathrm{VCoSb}, \mathrm{NbCoSb}$, and $\mathrm{TaCoSb}$ when the concentration of vacancies at the cationic site is increased. The method proved unambiguously that the systems are more stable at $20 \%$ of concentration and, according to the DFT calculations in the range $0-20 \%$, there is a metal-semiconductor transition. The transition in $\mathrm{VCoSb}$ includes a magnetic-nonmagnetic transition, while the other two systems remain non-magnetic. Further increase of the vacancies may lead to an $n$-doped semiconductor or to ingap states. The energy of formation and width of the gaps calculated in the three $\mathrm{HH}$ make us conclude that, at $20 \%$ of vacancy concentration, $\mathrm{Ta}_{0.8} \mathrm{CoSb}$ is the most stable, closely followed by $\mathrm{Nb}_{0.8} \mathrm{CoSb}$, while $\mathrm{V}_{0.8} \mathrm{CoSb}$ is the least stable of the three HHs.

This theoretical result predicts a slightly higher vacancy concentration than the experiments, where the stable stoichiometry of one of the compounds is found at $1 / 6$ vacancies. ${ }^{32}$ 
We should point out that the CE method deals simultaneously with the depletion of electrons and the distribution of them across the material as the amount of vacancies increases (see the Discussion for how the coordination number affects the chemical bonding of the system in Table I, and also the case of $\left.(\mathrm{V}, \mathrm{Nb}) \mathrm{TaCoSb}^{46}\right)$. It is worth noting that one expects a larger lattice distortion as the amount of vacancies increases. In this regard, although we included atomic relaxation for all the structures displayed in Fig. 2 in our DFT calculation, cooperative lattice distortions in extended systems may favor lower energies for $1 / 6$ than for $1 / 5$ of vacancies.

According to the combined information of our DFT and MC results, the choice of synthetization is crucial to obtain the maximum semi-conducting gap: only under very controlled methods can the most stable structures and corresponding gaps be delivered. However, other metastable structures with less refined synthetization methods should be fairly accessible, albeit with a reduced gap. The lower lattice thermal conductivity of defective HHs, and the close relationship between vacancy concentration and short-range order, ${ }^{47,48}$ strongly suggest the continuation of studies to establish optimal atomic arrangements ${ }^{49}$ which can lower the lattice thermal conductivity without compromising too much the electronic transport properties. In this sense, our results also show that heat treatment can be used to manipulate the amount of vacancy-ordered structures.

\section{Supporting Information}

Supporting Information is available from the Wiley Online Library or from the author.

Supplementary Information The online version contains supplementary material available at https://doi.org/10.1007/s11664-022-09510-0.

Acknowledgments Our research work is funded by the Deutsche Forschungsgemeinschaft (DFG, German Research Foundation) - Project Number 392228380. The calculations have been performed using the ab-initio total-energy and molecular-dynamics program VASP (Vienna ab-initio simulation program) developed at the Institut für Materialphysik at the Universität Wien. ${ }^{38,39}$

Funding Open Access funding enabled and organized by Projekt DEAL.

Conflict of interest The authors declare that they have no conflict of interest.

Open Access This article is licensed under a Creative Commons Attribution 4.0 International License, which permits use, sharing, adaptation, distribution and reproduction in any medium or format, as long as you give appropriate credit to the original author(s) and the source, provide a link to the Creative Commons licence, and indicate if changes were made. The images or other third party material in this article are included in the article's Creative Commons licence, unless indicated otherwise in a credit line to the material. If material is not included in the article's Creative Commons licence and your intended use is not permitted by statutory regulation or exceeds the permitted use, you will need to obtain permission directly from the copyright holder. To view a copy of this licence, visit http://creativecommons.org/licenses/by/4.0/.

\section{References}

1. W. von Ammon, A. Sattler, and G. Kissinger, Defects in Monocrystalline Silicon. (Springer, Cham, 2017), p. 1.

2. A. Walsh and A. Zunger, Nat. Mater. 16, 964 (2017).

3. S. Zhang, S.B. Ogale, W. Yu, X. Gao, T. Liu, S. Ghosh, G.P. Das, A.T.S. Wee, R.L. Greene, and T. Venkatesan, Adv. Mater. 21(22), 2282 (2009).

4. A. Janotti and C.G. Van de Walle, Phys. Rev. B 76, 165202 (2007).

5. A. Rusydi, S. Dhar, A.R. Barman, N. Ariando, D.-C. Qi, M. Motapothula, J.B. Yi, I. Santoso, Y.P. Feng, K. Yang, Y. Dai, N.L. Yakovlev, J. Ding, A.T.S. Wee, G. Neuber, M.B.H. Breese, M. Ruebhausen, H. Hilgenkamp, and T. Venkatesan, Philos. Trans. R. Soc. A 370, 1977 (2012).

6. K. Yim, Y. Youn, M. Lee, D. Yoo, J. Lee, S.H. Cho, and S. Han, npj Comput. Mater. 4, 17 (2018).

7. A. Page, C. Uher, P.F. Poudeu, and A. Van der Ven, Phys. Rev. B 92, 174102 (2015).

8. Y.G. Yu, X. Zhang, and A. Zunger, Phys. Rev. B 95, 085201 (2017).

9. C. Fu, M. Yao, X. Chen, L.Z. Maulana, X. Li, J. Yang, K. Imasato, F. Zhu, G. Li, G. Auffermann, U. Burkhardt, W. Schnelle, J. Zhou, T. Zhu, X. Zhao, M. Shi, M. Dressel, A.V. Pronin, G.J. Snyder, and C. Felser, Adv. Sci. 7(1), 1902409 (2020).

10. N.S. Chauhan, P.R. Raghuvanshi, K. Tyagi, K.K. Johari, L. Tyagi, B. Gahtori, S. Bathula, A. Bhattacharya, S.D. Mahanti, V.N. Singh, Y.V. Kolen'ko, and A. Dhar, J. Phys. Chem. C 124(16), 8584 (2020).

11. J. Shen, X. Zhang, S. Lin, J. Li, Z. Chen, W. Li, and Y. Pei, J. Mater. Chem. A 4, 15464 (2016).

12. G. Tan, W.G. Zeier, F. Shi, P. Wang, G.J. Snyder, V.P. Dravid, and M.G. Kanatzidis, Chem. Mater. 27(22), 7801 (2015).

13. S. Chen, H. Bai, J. Li, W. Pan, X. Jiang, Z. Li, Z. Chen, Y. Yan, X. Su, J. Wu, C. Uher, and X. Tang, ACS Appl. Mater. Interfaces 12(17), 19664 (2020).

14. S. Öğüt and K.M. Rabe, Phys. Rev. B 51, 10443 (1995).

15. D. Jung, H.-J. Koo, and M.-H. Whangbo, J. Mol. Struct. THEOCHEM. 527(1), 113 (2000).

16. J. Toboła and J. Pierre, J. Alloys Compd. 296(1), 243 (2000)

17. I. Galanakis, P.H. Dederichs, and N. Papanikolaou, Phys. Rev. $B$ 66, 134428 (2002).

18. H.C. Kandpal, C. Felser, and R. Seshadri, J. Phys. D Appl. Phys. 39(5), 776 (2006).

19. L. Offernes, P. Ravindran, C. Seim, and A. Kjekshus, J. Alloys Compd. 458(1), 47 (2008).

20. T. Graf, C. Felser, and S.S. Parkin, Prog. Solid State Chem. 39(1), 1 (2011).

21. F. Casper, T. Graf, S. Chadov, B. Balke, and C. Felser, Semicond. Sci. Technol. 27(6), 063001 (2012).

22. M. Zeeshan, H.K. Singh, J. van den Brink, and H.C. Kandpal, Phys. Rev. Mater. 1, 075407 (2017).

23. S.J. Poon, Metals 8, 989 (2018).

24. L. Huang, R. He, S. Chen, H. Zhang, K. Dahal, H. Zhou, H. Wang, Q. Zhang, and Z. Ren, Mater. Res. Bull. 70, 773 (2015).

25. L. Huang, Y. Wang, J. Shuai, H. Zhang, S. Yang, Q. Zhang, and Z. Ren, RSC Adv. 5, 102469 (2015). 
26. K. Xia, Y. Liu, S. Anand, G.J. Snyder, J. Xin, J. Yu, X. Zhao, and T. Zhu, Adv. Funct. Mater. 28, 1705845 (2018).

27. W.G. Zeier, S. Anand, L. Huang, R. He, H. Zhang, Z. Ren, C. Wolverton, and G.J. Snyder, Chem. Mater. 29, 1210 (2017).

28. L. Huang, J. Wang, X. Mo, X. Lei, S. Ma, C. Wang, and Q. Zhang, Materials 12, 1637 (2019).

29. S. Anand, K. Xia, V.I. Hegde, U. Aydemir, V. Kocevski, T. Zhu, C. Wolverton, and G.J. Snyder, Energy Environ. Sci. 11, 1480 (2018).

30. S. Anand, K. Xia, T. Zhu, C. Wolverton, and G.J. Snyder, $A d v$. Energy Mater. 8(30), 1801409 (2018).

31. S. Tan, P. Nan, K. Xia, H. Yang, T. Zhu, B. Ge, and W. Zhang, J. Phys. Chem. C 125(1), 1125 (2021).

32. N. Roth, J. Beyer, K.F.F. Fischer, K. Xia, T. Zhu, and B.B. Iversen, IUCrJ 8(Pt 4), 695 (2021).

33. S. Guo, K. Yang, Z. Zeng, and Y. Zhang, Phys. Chem. Chem. Phys. 20, 14441 (2018).

34. J. Miranda Mena and T. Gruhn, Phys. Rev. B 101, 064201 (2020).

35. J.M. Sanchez, Phys. Rev. B 81, 224202 (2010).

36. A. van de Walle, M. Asta, and G. Ceder, Calphad 26(4), 539 (2002).

37. P.E. Blöchl, Phys. Rev. B 50, 17953 (1994).

38. G. Kresse and J. Furthmüller, Phys. Rev. B 54, 11169 (1996).
39. G. Kresse and D. Joubert, Phys. Rev. B 59, 1758 (1999).

40. K. Kaczmarska, J. Pierre, J. Beille, J. Tobola, R. Skolozdra, and G. Melnik, J. Magn. Magn. Mater. 187(2), 210 (1998).

41. N. Metropolis, A.W. Rosenbluth, M.N. Rosenbluth, A.H. Teller, and E. Teller, J. Chem. Phys. 21(6), 1087 (1953).

42. J.M. Cowley, Phys. Rev. 77, 669 (1950).

43. D. De Fontaine, J. Appl. Crystallogr. 4(1), 15 (1971).

44. A.I. Gusev, Phys.-Uspekhi 49(7), 693 (2006).

45. A. Zunger, S.-H. Wei, L.G. Ferreira, and J.E. Bernard, Phys. Rev. Lett. 65, 353 (1990).

46. J.M. Mena and T. Gruhn, J. Mater. Chem. A 9, 21111 (2021).

47. K. Xia, P. Nan, S. Tan, Y. Wang, B. Ge, W. Zhang, S. Anand, X. Zhao, G.J. Snyder, and T. Zhu, Energy Environ. Sci. 12, 1568 (2019).

48. N. Roth, T. Zhu, and B.B. Iversen, IUCrJ 7(4), 673 (2020).

49. J. Miranda and T. Gruhn, Comput. Mater. Sci. 204, 111147 (2022).

Publisher's Note Springer Nature remains neutral with regard to jurisdictional claims in published maps and institutional affiliations. 